\title{
Lexikalische Typologie - Dänisch und Französisch als endo- bzw. exozentrische Sprachen
}

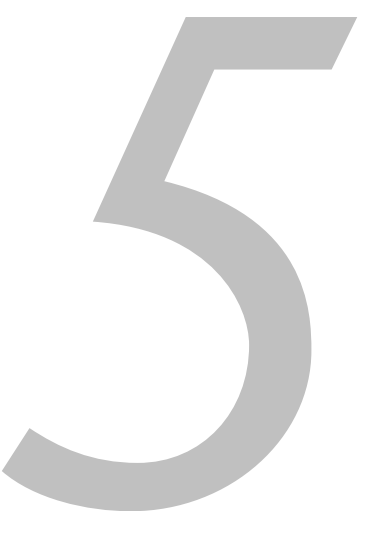

\author{
Michael HeRsLUND \\ CBS - Wirtschaftsuniversität Kopenhagen, Dänemark
}

\begin{abstract}
The article discusses one of the lesser studied aspects of linguistic typology, the lexicon. Taking as its point of departure the theory of lexicalisation of Leonard Talmy (1985), i.e. the different combinations of (presumably) universal semantic components different (types) of languages choose to code in their simple lexical roots, the article applies this approach to French and Danish typology is not just a question of comparing so-called exotic languages. It appears that just as verb roots in Germanic, such as Danish, and Romance languages, such as French, differ fundamentally in their lexicalisation patterns, so do their nouns. And more importantly, the two word classes exhibit complementary lexicalisation patterns: in Germanic languages the verbs are rather concrete - what is lexicalised is the external, visible aspect of the situation described by the verb, the manner - whereas the Romance verbs are abstract, describing i.e. the idea of a movement and its direction; in nouns, however, the situation is the exact opposite: the Germanic nouns are rather abstract, covering a wide range of phenomena having a certain function in common, but the Romance nouns are concrete, describing the external, visible aspect of things, their form rather than their function. This finding permits the hypothesis of two fundamental linguistic types: the endocentric languages (Germanic), where the concrete information is found in the centre of the clause, the verb, and the exocentric languages (Romance), where this information is found outside the centre, in the nouns.
\end{abstract}

Wenn man von linguistischer Typologie spricht, ist meistens von Morphologie und Syntax die Rede. Das Lexikon, das heißt der Wortschatz einer Sprache, wird manchmal als etwas Unsystematisches, eine zufällige Menge von arbiträren Bezeichnungen für mehr oder weniger universelle Begriffe angesehen.

Rijkhoff, Jan (ed.), Linguistic Typology, Århus: Statsbiblioteket

Tidsskrift for Sprogforskning, årgang 5, 2007

Artikel nr. 5, Herslund, Michael, 13 pp.

http://ojs.statsbiblioteket.dk/index.php/tfs/index 
Tatsächlich sind viele Linguisten der Meinung, dass die Wörter verschiedenen Sprachen dieselben Bedeutungen tragen: Übersetzungsäquivalente haben, so oder so, denselben Sinn. Für viele Linguisten, von Bloomfield bis Chomsky, ist das Lexikon einer Sprache nur das Inventar ihrer Idiosynkrasien und Unregelmäßigkeiten, was einer Auffassung entspricht, welche diametral entgegengesetzt der von Saussure ist. Für ihn erschien der Sinn (la valeur) eines Wortes von dem aller anderen Wörter der Sprache abgegrenzt, wodurch er festgelegt und definiert wird, ja überhaupt nur zu begreifen ist.

Ich mache mir die Auffassung von Saussure zu Eigen, und werde zu zeigen versuchen, dass das Lexikon einer Sprache auf der einen Seite sehr systematisch organisiert ist, und dass, auf der anderen Seite, die Sprachen nach ihrem lexikalischen Aufbau typologisierbar sind.

Der Anlaß meiner Überlegungen ist die folgende Äußerung von Roman Jakobson: "Languages differ essentially in what they must convey and not in what they may convey" (Jakobson 1959: 236). Obwohl Jakobson in diesem Artikel meist von Morphologie und Syntax und nur andeutungsweise vom Lexikon spricht, kann man ohne weiteres seine Beobachtung direkt auf die lexikalische Ebene beziehen. Man kommt dann zu der Formulierung, dass eine Sprache nicht vermeiden kann auszudrücken, was in ihren einfachen, das heißt nicht zusammengesetzten oder derivierten, Lexemen bereits kodiert, oder lexikalisiert ist - wenn sie auch alles sonst durch Derivation, Komposition, Phraseologismen, verschiedene syntaktische Konstruktionen, usw. ausdrücken kann.

Daraus folgt auch, dass alle Sprachen, eben weil sie alles ausdrücken können, in gewisser Weise dieselbe Komponente besitzen, dass sie alle sozusagen Zugang zu einem gemeinsamen, universellen Vokabular haben. Da die Sprachen also sehr vieles gemeinsam haben, und sich dennoch dadurch unterscheiden, was sie ausdrücken müssen, wird es zunächst notwendig sein, verschiedene Ebene zu unterscheiden.

Wir können in einem ersten Schritt eine universelle, kognitiv angelegte Ebene annehmen, wo wir die semantischen Komponenten (oder Atome $\{a, b, \ldots z\}$ ) finden, die allen Sprachen gemeinsam sind: die Universalebene (vgl. Talmy 
1985, 2000). In einem zweiten Schritt nehmen wir an, dass die verschiedenen Sprachen jeweils eigene Kombinationen dieser Komponenten auswählen, z.B. \{a $+\mathrm{c}\}$ oder $\{a+d\}$, usw., um ihre Lexeme zu bilden: die typologische Ebene, wo die Sprachen sich in Typen gemäß der vorgezogenen Kombinationen gruppieren, von $T_{1}$ bis $T_{n}$. Auf der untersten Ebene haben wir dann die einzelnen individuellen Sprachen mit allen ihren Idiosynkrasien, vgl. Abbildung 1 und Herslund (2003).

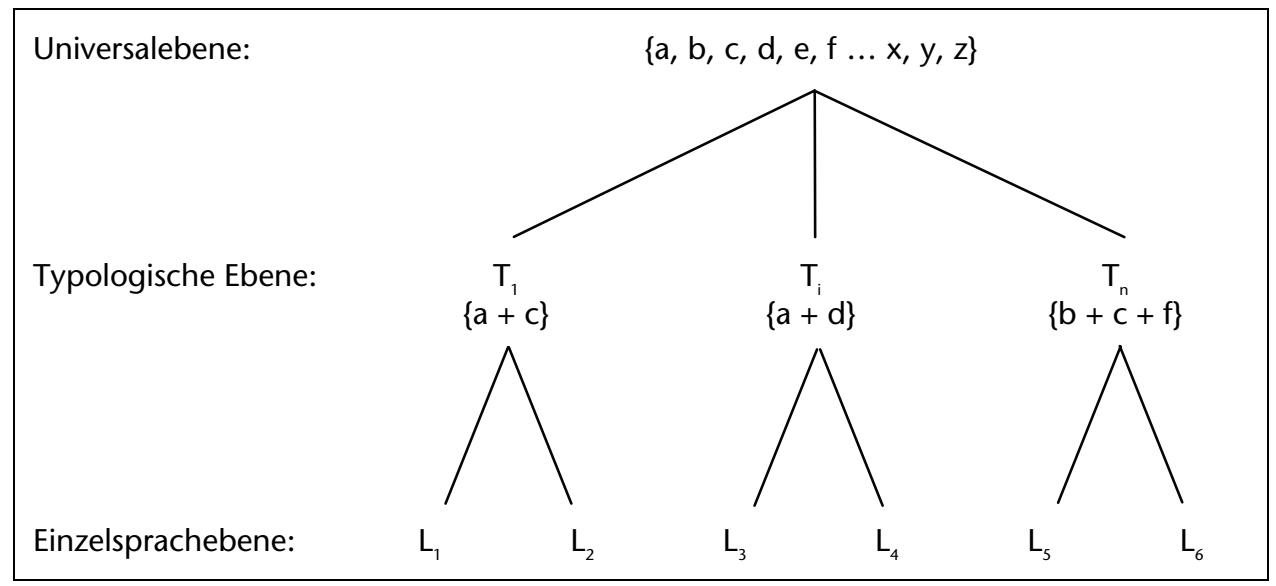

Abb. 1

Ich werde in meinem Beitrag zwei Sprachen, Französisch und Dänisch - die Typologie muss nicht "exotisch" sein! -, im Lichte dieser Auffassung vergleichen und obiges Schema konkretisieren. Mein Ausgangspunkt sind Überlegungen von Leonard Talmy $(1985,2000)$ und seine Analyse von Bewegungsverben als Beispiel für das, was Talmy "Lexikalisierung" nennt, das heißt die Bildung von einfachen Lexemen durch semantische Komponenten. Für unsere Analyse benötigen wir nur drei der fünf von Talmy vorgeschlagenen universellen semantischen Komponenten, welche für die Beschreibung eines beliebigen Bewegungsverbs notwendig und zureichend sind. Diese Komponenten sind:

- die Bewegung als solche - BEWEGUNG

- die Richtung und das Ziel der Bewegung - RICHTUNG

- die Art und Weise der Bewegung - ART 
Wenn wir jetzt die französischen und dänischen Bewegungsverben im Lichte dieser Komponenten analysieren, werden wir sehen, dass die Verben dieser zwei Sprachen - und im Weiteren die Verben der romanischen und der germanischen Sprachen generell - sich systematisch unterscheiden, und zwar dadurch, dass die normalen französischen Verben, die ausdrücken, was Tesnière (1959) déplacement nennt, die Komponente RICHTUNG enthalten, während die dänischen Verben einer solcher Komponente entbehren; dänische Verben besitzen dafür immer eine Komponente ART, und müssen damit, ob sie wollen oder nicht, die Art der Bewegung ausdrücken.

Um eine Bewegung vorwärts zu beschreiben, nutzt das Französische das Verb avancer, ohne die Art der Bewegung zu präzisieren. Eine solche Präzisierung kann zum Beispiel durch eine Ergänzung in der Form eines "gérondifs" (wie z. B. en nageant 'schwimmend') oder eines ähnlichen Adverbialausdrucks (wie z. B. à la nage 'durch Schwimmen') stattfinden, muss es aber keinesfalls:

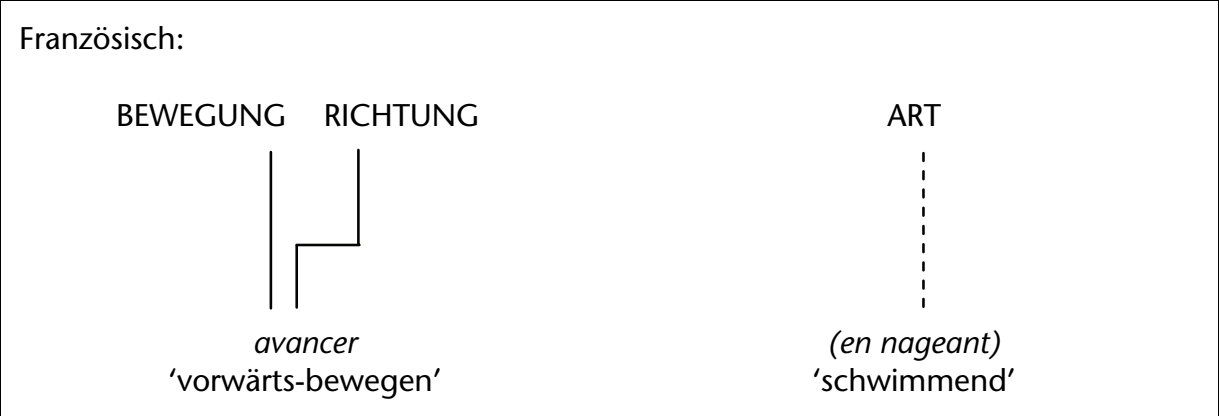

Abb. 2

Das Verb avancer bildet mit anderen zielbezogenen Verben wie aller, venir, entrer, sortir, monter, descendre, reculer usw. die sogenannte inakkusativische Verbalklasse des Französischen, vgl. Herslund (1990).

Um dieselbe Situation im Dänischen auszudrücken, kann man, wie im Deutschen, nur ein Verb wählen, das - wie etwa schwimmen, gehen, laufen usw. nicht nur eine Bewegung sondern auch ihre Art und Weise ausdrückt: 
Dänisch:

BEWEGUNG ART

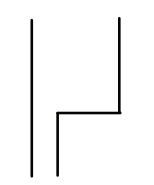

svømme

gå

lobe

flyve

$\ldots$

\section{RICHTUNG}

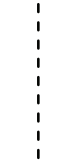

(fremad)

'schwimmen, gehen, laufen, fliegen ...'

'vorwärts'

Abb. 3

Wenn man auch die Richtung ausdrücken will - ohne das aber ausdrücklich zu müssen - kann das nur durch eine Ergänzung in der Form eines Adverbs wie fremad 'vorwärts' oder eines Präfixes geschehen.

Was also in den einfachen Verballexemen einer Sprache nicht vorhanden ist, kann, muss aber nicht, außerhalb der Lexemgrenzen ausgedrückt werden:

- Im Französischen wird die Richtung einer Bewegung vom Verb selbst ausgedrückt, ihre Art und Weise dagegen durch die Hinzufügung eines Verbum infinitum, eines Adverbials oder ähnlichem, spezifiziert, vgl. Abb. 2. Das Französische ist also, was Talmy (2000) eine 'verb framed' Sprache nennt.

- Im Dänischen wird die Richtung durch die Hinzufügung eines Adverbs oder eines Präfixes ausgedrückt, vgl. Abb. 3. Das Dänische erweist sich damit als eine 'satellite framed' Sprache (ib.).

Freilich hat auch das Französische Verben, die die Art und Weise einer Bewegung ausdrücken, bzw. nager 'schwimmen', marcher 'gehen', courir 'laufen' usw., aber diese Verben sind unfähig, gleichzeitig die Richtung einer Bewegung auszudrücken. Diese Verben können also nicht ein déplacement bezeichnen. Der große Unterschied zwischen den beiden Sprachen ist, dass das Dänische nur eine Klasse von Verben besitzt, während das Französische zwei Serien von Bewegungsverben hat: die inakkusativischen Verben wie avancer, aller, sortir, venir, entrer usw., und die sogenannten inergativen Verben wie nager, marcher 
usw., vgl. Herslund (1990, 2003). Die letztgenannten Verben drücken nie die Richtung oder das Ziel aus (RICHTUNG), und um diese Information auszudrücken, muss man richtungsdenotierende Präpositionalausdrücke wie jusqu'à 'bis zu' oder ähnliches hinfügen. Sowohl diese Verben als auch die einfachen Präpositionen wie à, en, dans usw. sind unfähig, diese Komponente wiederzugeben:

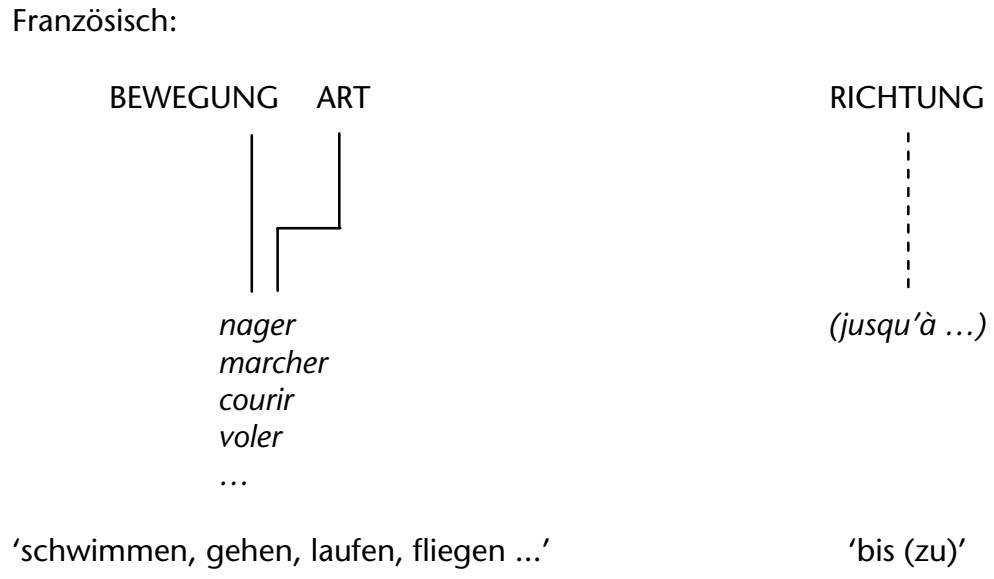

Abb. 4

Ich habe an anderer Stelle (Herslund 2003) den Vergleich der beiden Sprachen mit ausführlichen Übersetzungsbeispielen illustriert.

Was nun für die Typologie wichtig ist, ist, dass die Tatsache, dass die dänischen Bewegungsverben immer die Komponente ART ausdrücken, auf viele andere Verben aus anderen semantischen Feldern generalisierbar ist, vgl. Schema 1:
1. gå, løbe, springe, hoppe...
aller

Wie schon gezeigt, muss das Dänische die Art und Weise einer Bewegung beschreiben, während das Französische nur die Richtung präzisiert. Das einfache Verb aller drückt genau so eine zielbezogene Bewegung (déplacement) aus, ohne die Art und Weise der Bewegung zu nennen. 
LEXIKALISCHE TYPOLOGIE - DÄNISCH UND FRANZÖSISCH ALS ENDO- BZW. EXOZENTRISCHE SPRACHEN

\begin{tabular}{|l|l|}
\hline 2. stå, sidde, ligge & ettre \\
\hline $\begin{array}{l}\text { Wie mit der Bewegung im Dänischen ist es auch mit der einfachen Ruheposition: Die } \\
\text { Art der Position muss ausgedrückt sein, wie im Deutschen bei Verben wie stehen, } \\
\text { sitzen, liegen. Im Französischen reicht schon ein einfaches être 'sein'. }\end{array}$
\end{tabular}

\begin{tabular}{l|l} 
3. stille, scette, loegge & mettre
\end{tabular}

Vergleichbar sind die Verhältnisse, wenn eine solche Ruheposition das Ergebnis einer Anbringung eines Objekts ist: Im Dänischen muss man wieder die Position eines platzierten Objektes präzisieren, also ob man es stellt, setzt oder legt. Im Französischen genügt ein einfaches Verbum mettre im Sinne von 'anbringen'.

\begin{tabular}{|l|l|}
\hline 4. skcere, save, hugge, klippe ... & couper \\
\hline Um ein Objekt in zwei Teile zu zerlegen, muss das Dänische die Art der Zerlegung \\
nennen, ob man schneidet, sägt, haut oder mit einer Schere schneidet. Im \\
Französischen gibt es das generelle Verb couper 'zerlegen' - und die französische \\
Sprache braucht wieder - wie immer - nicht die Art des Vorgehens zu nennen.
\end{tabular}

5. broekke, knokke, smadre...

casser

Um ein Objekt zu zerstören, muss man im Dänischen darüber hinaus die Art und Weise präzisieren (brechen, reißen, zerschmettern usw.). Im Französischen reicht das einfache casser.

\begin{tabular}{|l|l|}
\hline 6. stikke, hugge, slå, skyde ... & frapper \\
\hline $\begin{array}{l}\text { Im Französischen bedeutet frapper 'treffen (mit einer Waffe)', im Dänischen muss } \\
\text { man nach der Art der Waffe präzisieren (stechen, hauen, schlagen, schießen, usw.). }\end{array}$ \\
\hline
\end{tabular}

\begin{tabular}{|l|l|}
\hline 7. koge, stege, bage ... & cuire \\
\hline $\begin{array}{l}\text { Während das Französische das einfache Verb cuire 'Essen mit Hilfe von Wärme } \\
\text { zubereiten' besitzt, muss man im Dänischen wiederum die Art der Zubereitung } \\
\text { ausdrücken (kochen, braten, backen ...). Natürlich kann das Französische diese } \\
\text { verschiedenen kulinarischen Einzelheiten versprachlichen und sehr eingehend } \\
\text { präzisieren, muss es aber nicht! }\end{array}$
\end{tabular}


Technologische Vortschritte haben zum Beispiel die Konsequenz, dass die Dänen, die von Telefonen mit Wählscheiben zu Apparaten mit Drucktasten wechseln, auch Verb wechseln müssen, eben weil das dänische Verb immer die ART ausdrückt: Um einen Nummer zu wählen, muss man drehen oder drücken. Die Franzosen 'komponieren', ohne sich weitere Sorgen über das richtige Verb zu machen, einfach die Nummer: composer. Das Verb bleibt also auch weiterhin brauchbar unabhängig vom Typ des Gerätes, weil es die ART nicht ausdrückt.

\section{Schema 1}

Was hier wichtig ist einzusehen, ist, dass das Französische in allen diesen Fällen dasselbe wie das Dänische ausdrücken kann: Man kann natürlich 'stehen', 'stellen', 'sägen', usw. in dieser Sprache. Aber das Dänische kann es nicht vermeiden, diese Information mitzugeben.

Wie ist es dann mit der anderen Hauptwortklasse, den Substantiven? Das Hauptmuster ist hier, wie es im Schema 2 dargestellt ist: 


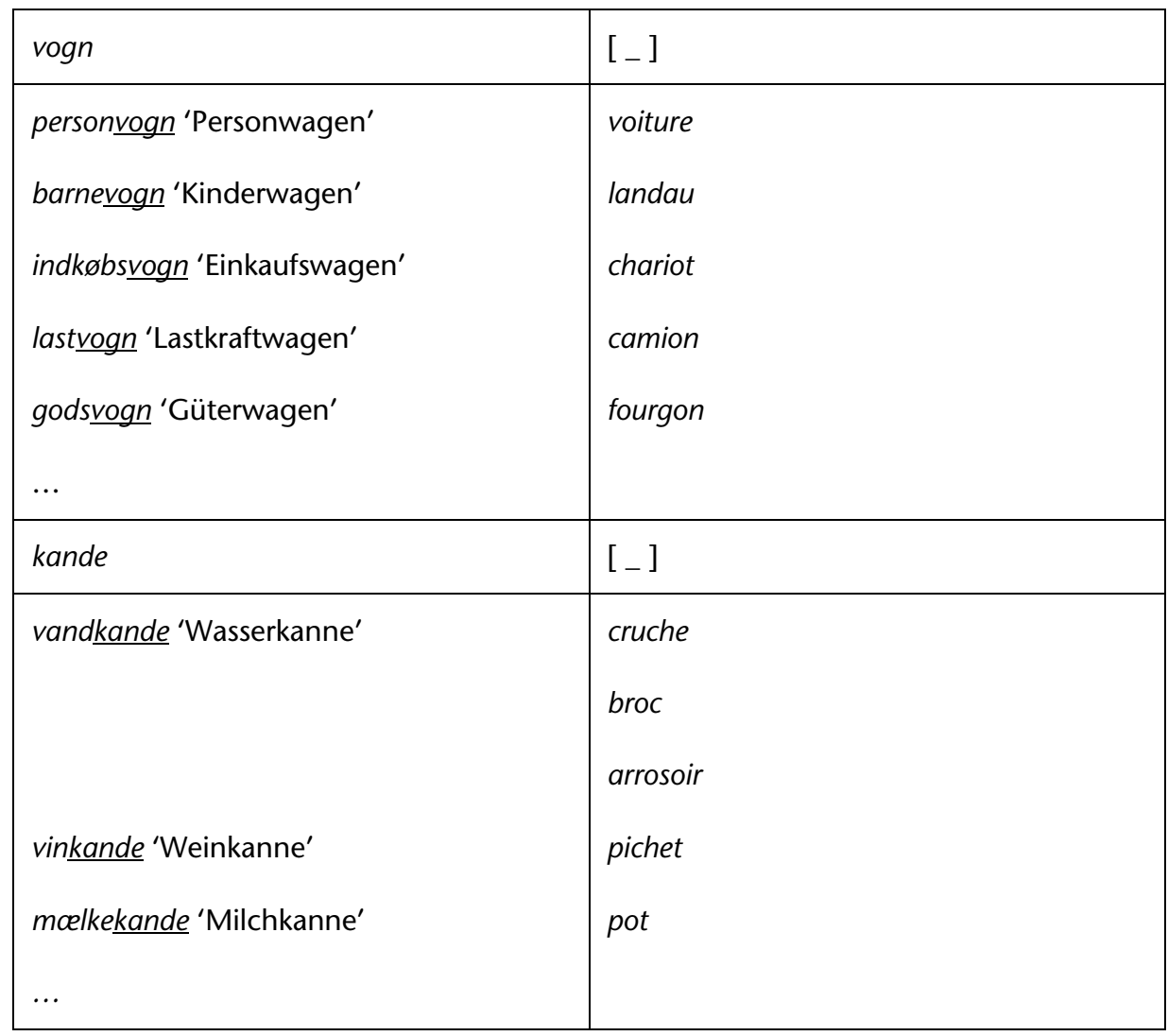

\section{Schema 2}

Diese Verhältnisse sind folgendermaßen zu analysieren (vgl. Baron 2003, Baron \& Herslund 2005):

- Im Dänischen finden wir ein Wort (ein Lexem), in unseren Beispielen vogn 'Wagen' und kande 'Kanne', das in verschiedenen Zusammensetzungen wiederkehrt - wie im Deutschen. Das Hauptmuster besteht dann aus einem Hyperonym und einer Serie von Ko-Hyponymen, die durch Komposition mit dem Hyperonym gebildet sind.

- Im Französischen dagegen haben wir normalerweise eine Serie von morphologisch nicht verwandten Wörtern, die nicht von einem einzelnen Hyperonym abhängen und ebenso wenig ableitbar sind.

Im Dänischen finden wir dann sehr generelle Substantive, das heißt Substantive mit kleiner Intension, aber einer großen Extension: Das Wort vogn 'Wagen' denotiert die sehr große Klasse von allem, was sich auf mehr als zwei Rädern 
bewegt und zum Transport von Personen oder Sachen bestimmt ist; das Wort kande 'Kanne' bezeichnet alle Objekte, welche zum Ausgießen von Flüssigkeiten bestimmt sind. Diese Substantive können dann durch Zusammensetzungen präzisiert werden, müssen es aber nicht. Diese Präzisierung hat den Charakter der Bildung einer Serie von Hyponymen. Obwohl auch die französischen Substantive der Serie \{cruche, broc, arrosoir, pichet, pot\}, vgl. Schema 2, ein semantisches Feld bilden, kann man eben nicht von Hyponymen oder KoHyponymen reden, weil ihnen ein gemeinsames Hyperonym fehlt, was die leeren eckigen Klammern der in Schema 2 erklärt.

Im Französischen dagegen finden wir schematisch sehr enge und präzise Wörter mit großer Intension, aber einer kleinen Extension: Das Wort pichet '(Wein)kanne', zum Beispiel, bezeichnet nur eine besondere Art von Kannen und Kännchen, die auch alles andere als Wein enthalten können; das Wort chariot 'Einkaufswagen, Kofferkuli' denotiert lediglich die beschränkte Klasse sehr spezieller Einrichtungen, die man nur in Kaufhäusern und Flughäfen findet.

Wenn wir jetzt die Idee der semantischen Komponenten auch für die Substantive nutzen wollen, sehen wir sofort, dass die Hauptkomponente der dänischen Substantive die Funktion des Objektes denotiert, während die Hauptkomponente im Französischen darauf fokussiert, wie der Gegenstand ausgeformt ist, wie er aussieht, also seine Konfiguration. Die Bezeichnungen 'Funktion' und 'Konfiguration' entlehne ich der sogenannten Qualia-Struktur von James Pustejovsky (1995).

Was wir dann feststellen können, ist also eine "umgekehrte" und komplementäre Verteilung der semantischen Komponenten auf die zwei Hauptwortklassen:

- In den Verben kodiert das Dänische die Art und Weise, das heißt das Aussehen, den sichtbaren Aspekt der Verbalsituation, während das Französische etwas Abstrakteres, nicht unmittelbar Sichtbares wie die Richtung oder das Ziel einer Bewegung kodiert.

- Bei den Substantiven sind die Verhältnisse umgekehrt: Die Funktion eines Objektes ist im Dänischen nicht unmittelbar sichtbar, ist abstrakt. Die Konfiguration eines Objektes dagegen ist unmittelbar wahrnehmbar und konkret, und das ist, was das Französische kodiert. 
Im Gebet der Substantive ist das Dänische also abstrakt, das Französische dagegen sehr konkret. Vgl. Schema 3 und 4:

\begin{tabular}{|l|c|c|}
\hline & ART & RICHTUNG \\
\hline Französisch: avancer & - & + \\
\hline Dänisch: gå ... & + & - \\
\hline
\end{tabular}

Schema 3

\begin{tabular}{|l|c|c|}
\hline & KONFIGURATION & FUNKTION \\
\hline Französisch: pichet & + & - \\
\hline Dänisch: kande & - & + \\
\hline
\end{tabular}

Schema 4

Wir können also eine typologische Skala wie die folgende annehmen (Abb. 5), indem wir unter Wiederverwendung, oder, genauer gesagt, Umdefinition der bekannten Bloomfieldschen syntaktischen Terme die zwei verschiedenen Typen "endozentrisch" bzw. "exozentrisch" nennen:

\begin{tabular}{|c|c|}
\hline Endozentrische Sprachen & Exozentrische Sprachen \\
\hline $\begin{array}{l}\text { spezifische Verben } \\
\text { generische Substantive }\end{array}$ & $\begin{array}{l}\text { generische Verben } \\
\text { spezifische Substantive }\end{array}$ \\
\hline Dänisch & Französ \\
\hline
\end{tabular}

Abb. 5

Es handelt sich hier um eine Skala; verschiedene Sprachen sind damit mehr oder weniger nah an den äußeren Rändern der Skala zu platzieren: Während das Dänische und das Französische sicherlich zwei Extremen bilden, ist eine romanische Sprache wie das Italienische mehr "gemischt" (vgl. Herslund 2005) und das Englische würde einem Typ entsprechen, der im Vergleich mit den anderen germanischen Sprachen verhältnismäßig viele exozentrische Züge aufweist (vgl. Baron \& Herslund 2005). 
Die zwei extremen Beispiele sind dann folgendermaßen zu erklären:

- Dänisch ist endozentrisch, weil die konkrete, spezifische lexikalische Information im Zentrum des Satzes - im Verb - kodiert ist, vgl. Abb. 6.

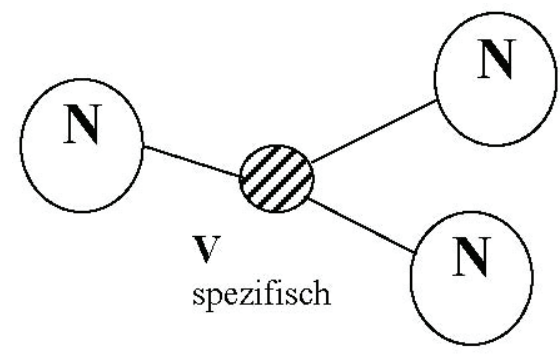

Abb. 6: Endozentrische Sprache

- Französisch ist exozentrisch, weil die konkrete und spezifische lexikalische Information an der Peripherie des Satzes - in den Substantiven - kodiert ist, vgl. Abb. 7.

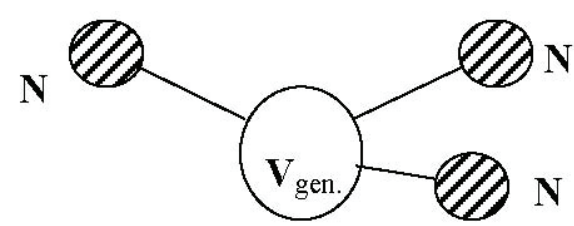

Abb. 7: Exozentrische Sprache

Die Forschungsgruppe für typologische Linguistik und Übersetzung (TYPOlex) an der Wirtschaftsuniversität Kopenhagen hat ferner auch eine Reihe grammatischer und textueller Konsequenzen dieser lexikalisch-typologischen Grundlage untersucht, wie sie zum Beispiel in den gemeinsamen Publikationen von I. Korzen \& Marello, Hrsg. (2000), M. Herslund, Hrsg. (2003), I. Korzen \& L. Lundquist, Hrsg. (2003), I. Baron, Hrsg. (2005), M. Herslund \& I. Baron, Hrsg. (2005), und I. Korzen \& P. D’Achille, Hrsg. (2005) dargestellt sind. 


\section{LITERATUR}

Baron, Irène (2003), "Catégories lexicales et catégories de pensée : une approche typologique du danois et du français", in: Herslund, Michael (Hrsg.) (2003), pp. 29-53.

Baron, Irène, (ed.) (2005), Language and Culture, Copenhagen Studies in Language 29.

Baron, Irène, Michael Herslund (2005), "Langues endocentriques et langues exocentriques. Approche typologique du danois, du français et de l'anglais", in: Herslund, Michael, Irène Baron (ed.) (2005), pp. 35-53.

Herslund, Michael (1990), "Les verbes inaccusatifs comme problème lexicographique", Cahiers de lexicologie 56-57, pp. 35-44.

Herslund, Michael, (ed.) (2003), Aspects linguistiques de la traduction, Bordeaux: Presses Universitaires de Bordeaux.

Herslund, Michael (2003), "Pour une typologie lexicale", in: Herslund, Michael (ed.) (2003), pp. 13-27.

Herslund, Michael (2005), "Lingue endocentriche e lingue esocentriche: aspetti storici del lessico", in: Korzen, I. \& P. D'Achille (ed.) (2005), pp. 19-30.

Herslund, Michael, Irène Baron, (ed.) (2005), Le génie de la langue française. Perspectives typologiques et contrastives, Langue française 145.

Jakobson, Roman (1959), "On Linguistic Aspects of Translation”, in: Bowers, R. A. (ed.), On Translation, Cambridge Mass.: Harvard University Press, pp. 232239.

Korzen, Iørn, Carla Marello, (ed.) (2000), Argomenti per una linguistica della traduzione, Gli argomenti umani 4, Alessandria: Edizioni dell'Orso.

Korzen, Iørn, Lita Lundquist, Hrsg. (2003), Sprogtypologi og oversattelse. Endocentriske og exocentriske sprog, København: Handelshøjskolen i København.

Korzen, Iørn, Paolo D’Achille, (ed.) (2005), Tipologia linguistica e società, Firenze: Franco Cesati.

Pustejovsky, James (1995), The Generative Lexicon, Cambridge Mass.: The MIT Press.

Talmy, Leonard (1985), "Lexicalization Patterns: Semantic Structure in Lexical Forms", in: Shopen, Timothy (ed.) (1985), Language Typology and Syntactic Description, Vol. III, pp. 57-149, Cambridge: The University Press.

Talmy, Leonard (2000), Toward a Cognitive Semantics, Vol. I-II, Cambridge, Mass.: The MIT Press.

Tesnière, Lucien (1959), Eléments de syntaxe structurale, Paris: Klincksieck. 